\title{
The way forward at Diabetes UK
}

\author{
CHRIS ASKEW
}

\section{Introduction}

To start with a brief introduction, I succeeded Barbara Young as Chief Executive of Diabetes UK last September, arriving from the breast cancer research and support charity, Breakthrough Breast Cancer, where I had worked for eight years, initially as Director of Fundraising and then as Chief Executive, leading the charity into a merger with a smaller breast cancer charity to form Breast Cancer Now. Before that, I held an interim role at JDRF, which marked my entry into the health sector.

Since arriving at Diabetes UK, I have understood the immense challenges of diabetes, at an individual and system level, but also the unique opportunity offered by the broader recognition of these challenges by politicians, health service leaders, healthcare professionals, people with diabetes, the public and the media. Our recent news release that the numbers of people with diabetes in the UK population has topped 4 million for the first time attracted huge attention. Public debate around addressing our obesogenic environment appears to be coalescing to a consensus that now is the time to take action. The National Diabetes Prevention Programme should be acknowledged as a first nation-wide investment in applying evidence to prevent the growing tide of Type 2 diabetes and it is right that Diabetes UK is central in driving this work forward. But there is much more yet to achieve.

The findings of November's National Audit Office report on adult diabetes services, re-emphasised in a Public Accounts Committee's review earlier this year, make for a sobering read; whilst there's been progress in lowering additional risk of death from diabetes (in truth, a change which has come about as a result of improvements in delivering the nine recommended care processes and achieving three treatment standards in the late 90s and early 2000s), there is little progress reported in other key areas since the initial report of 2012.

In the words of the report itself "significant geographic variations persist in the quality of care for people with diabetes and in their outcomes...", "some groups of diabetes patients, such as patients with Type 1 diabetes, receive worse routine care and have poorer outcomes....", "the percentage of hospital beds occupied by diabetes patients is increasing but levels of diabetes specialist staff have not changed... ", "current payment mechanisms within the NHS are

Diabetes UK, London, UK.

Address for correspondence: Chris Askew

CEO Diabetes UK, Camden Parkway, London, NW1 7AA, UK.

E-mail: Chris.askew@diabetes.org.uk

Br J Diabetes 2016:16:2-3

http://dx.doi.org/10.15277/bjd.2016.065

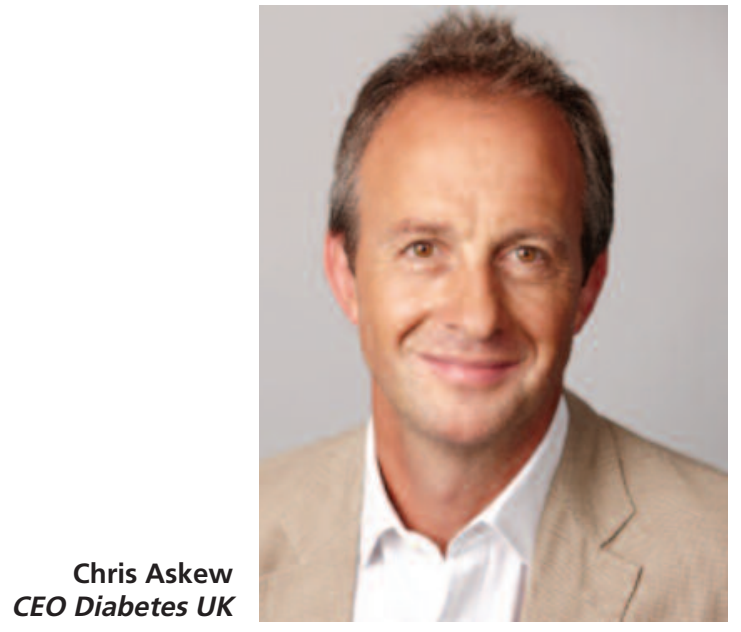

not offering incentives to integrate diabetes care .... ", "few people with diabetes are recorded as receiving patient education... ".

The more recent National Diabetes Audit looks set to report improved blood pressure across Type 1 and Type 2, but highlights the lowest level of completion of the eight care processes, since at least 2009, at 38.7\% and 58.7\% for Type 1 and Type 2 respectively.

I know that these issues concern us all hugely; I feel strongly that Diabetes UK's role, alongside highlighting the problems, is to work in partnership with specialists and others in the system, to develop effective solutions that help to change the picture for diabetes care. In this article I want to highlight some of the key challenges that stand out from my initial conversations with diabetologists and to outline some of the work that can help us, together, to grasp the opportunities to improve diabetes care.

\section{Fragmentation of care}

How many of you are working in hospitals that have patients from multiple clinical commissioning groups (CCGs), each with different expectations or commissioning arrangements for diabetes care? One of the most frustrating things when we look at the national picture of diabetes care is the endless re-inventing of wheels as each health board, CCG or equivalent develops its own diabetes solutions. Yes, services need to be responsive to local issues such as ethnic make-up, deprivation and urban vs rural populations, but that does not mean that we need to start with a blank sheet of paper when investigating how to improve care. Too often we see immense amounts of effort wasted as services are developed in isolation. To counter this our Shared Practice team have been scouring the country to identify examples of good practice that can be used to give you a head start 
when improving care. Explore the resources available at www. diabetes.org.uk/shared-practice

\section{Decision making divorced from clinical leadership}

A message that I hear very clearly from Specialist clinicians is that the current decision making structures work to exclude them from influencing the way that services are designed. Too often misunderstandings about the "purchaser/provider split" result in service re-designs excluding diabetes Specialists on the grounds that they represent the "provider", without considering their capacity to represent an expert voice in the delivery of diabetes care. To empower more clinical leaders we are currently entering the third year of supporting Diabetes UK Clinical Champions - a programme designed to give expert clinicians the skills and support they need to become effective leaders in diabetes care, challenge poor decision making and learn from national best practice. Recent successes of Clinical Champions in their local health economies have included improving access to new therapies; challenging cuts to pump funding; new guidelines to reduce hypos in older patients; and increasing specialist in-reach to inpatient care. To find out more about the programme visit www.diabetes.org.uk/clinical-champions-networks

\section{Variation in outcomes}

We are uniquely privileged in diabetes care to have the National Diabetes Audit which gives us a clear picture of the quality of care and allows us to see both that good care is possible - even in the currently stretched NHS - and that there are areas falling a long way short. Improving outcomes is a collaborative piece that starts with local health economies acknowledging that diabetes care is a problem and then developing effective improvement mechanisms. At Diabetes UK we will lobby both nationally and locally to persuade decision makers of the importance of focussing on and investing in the improvement of diabetes outcomes. We can also work with you as Specialists to ensure that the right resources are in place to drive improvement; everything from joint position statements that establish agreed best practice, to enabling the development of tomorrow's Specialists through our support of the Young Diabetologists and Endocrinologists Forum.

We also understand that you are operating as part of a wider health system and that we need to support that whole system to focus on improving outcomes. Our innovative information prescriptions can play a part in this; impacting both clinical and patient behaviour in a way that can improve key outcomes. Early research shows they are:
- Changing clinical behaviour - the information prescriptions automatically alert clinicians to patients who have diabetes and are outside the NICE targets for blood pressure, $\mathrm{HbA}_{1 \mathrm{c}}$ or cholesterol. We have case studies that show that this is prompting clinicians to intervene more proactively and identify patients who have slipped through the net for many years.

- Increasing patient understanding of key concepts - the information prescriptions are written in plain English and case studies show that many patients say the information prescription was the first time anyone explained their condition to them (clinicians are clear that it is not, but evidently the information prescription was the first explanation that hit home).

- Prompting patient action - anecdotally we are hearing of patients significantly improving key outcomes. The information prescriptions include a unique link to more information on Diabetes UK's website; we know that over 13,000 patients in 2015 have been sufficiently motivated by the receipt of an information prescription to access more information on managing their diabetes, showing that they are working as a prompt to action.

To find out more about the application of information prescriptions visit: www. diabetes.org.uk/info-p-qa

\section{Better support to self-care}

Whilst patients being better able to self-care is increasingly recognised as a "no-brainer" to improve outcomes and reduce costs, there is still a lack of action to increase the skills and capacity for self-care. Our latest campaign, Taking Control, aims to increase the availability and quality of diabetes education and to raise the demand from people with diabetes. Over 2016 we will continue to raise awareness of what diabetes education is, why it's important, and that people should have access to local courses. We will share more real people's experiences of going on courses and how these improved their lives. The campaign is supported by practical resources including a range of products for healthcare professionals and commissioners available here: www.diabetes.org.uk/self-management-education

As the focus across the UK shifts towards improving diabetes care, we have a unique opportunity to drive tangible change for people with diabetes. It is not something that any of us can do alone, but through building strong and effective partnerships with people with diabetes, government decision makers, commissioners and clinicians, I do commit Diabetes UK to playing a central role in improving diabetes care.

\section{Conflict of interest None. Funding None.}

